\title{
Ultrastructural and Molecular Characterization of Sarcocystis Species Derived from Macroscopic Sarcocysts of Domestic Sheep and Goats in Soran City, Erbil, Iraq
}

\author{
Sara Omar Swar ${ }^{1 *}$ and Bushra Hussain Shnawa ${ }^{2,3}$ \\ ${ }^{I}$ College of Agricultural Engineering Sciences, Salahaddin University, Kurdistan, Iraq \\ ${ }^{2}$ Department of Biology, Faculty of Sciences, Soran University, Kurdistan, Iraq \\ ${ }^{3}$ Scientific Research Center, Soran University, Kurdistan, Iraq \\ *Corresponding author's Email: sara_swar@yahoo.com; (D) ORCiD: 0000-0002-9977-932X
}

\begin{abstract}
This study aimed to identify Sarcocystis species isolated from macroscopic sarcocysts from naturally infected domestic sheep and goats using the molecular method, as well as investigating the morphological and the ultrastructural characteristics of the isolated species. A total of 1000 esophagi were collected from sheep and goats and examined for the presence of sarcocysts. Macroscopic sarcocysts were isolated from the infected esophagi, and Sarcosystis species were identified molecularly by $18 \mathrm{~S}$ rRNA gene sequence analysis. Moreover, the ultrastructure of the sarcocysts was investigated by both scanning and transmission electron microscopy. The macroscopic sarcocysts were detected in $9.1 \%(91 / 1000)$ of the esophagi. The results of electron microscopy indicated the characteristic features of the macroscopic sarcocysts. The cysts contained numerous merozoites and bananashaped bradyzoites. The bradyzoites were characterized by possessing a double-membrane pellicle and consisted of a conoid in one of the apices, numerous micronemes, two rhoptries, as well as a long, convoluted mitochondrion, subterminal nucleus, and several amylopectin granules. The partial analysis of the $18 \mathrm{~S}$ rRNA gene presented that all isolates produced bands of expected sizes on gel electrophoresis. The findings from the phylogenetic analysis revealed that the identified Sarcocystis species were most closely related to S. gigantea, $S$. moulei, and $S$. medusiformis. To the authors' knowledge, this is the first time $S$. medusiformis has been recorded in goats. Goats and sheep can be proposed as alternative intermediate hosts for S. gigantea and S. moulei, respectively, crossinfection may also occur between them and the host specificity of these species of Sarcocystis is questionable.
\end{abstract}

Keywords: Goats, Phylogeny, Sarcocystis, Sheep, Ultrastructure, 18S rRNA

\section{INTRODUCTION}

Sarcocystosis is a zoonotic protozoal disease that may cause mortality in many species of domestic and wild animals. Up to date, more than 196 valid species of Sarcocystis have been identified (Dubey et al., 2016). This parasite has an obligatory two-host life cycle, asexual stages develop in the intermediate host, which is frequently a herbivores animal, while sexual stages occur in the definitive host that is a carnivore or omnivore animal (Lindsay and Dubey, 2020). There are two sizes of Sarcocystis cysts, the microscopic and the macroscopic sarcocysts (Dubey, 2015). Several species of Sarcocystis develop macroscopic sarcocysts in the tissue of domesticated animals. Among them, S. caprafelis (S. moulei) in goats, S. gigantea (S. ovifelis), and S. medusiformis in sheep (Lindsay and Dubey, 2020). Two kinds of macroscopic sarcocysts are identified and classified as fat and thin in sheep. They represent S.gigantea and S. medusiformis, respectively (Farhang- Pajuh et al., 2014). In Iraq, a couple of different sizes of macroscopic Sarcocystis were recognized in the domestic goats. Fat sarcocysts appear to be large, ovoid, and only found in the esophageal muscle. Besides, the thin sarcocysts are less frequent and appear small and slender, and also are found in the diaphragm and skeletal muscle (Barham et al., 2005).

Traditionally, the ultrastructural characteristics of the Sarcocystis are considered as the fundamentals for the identification of various Sarcocystis spp. within the same intermediate host (Hu et al., 2017; Huang et al., 2019). Nowadays, genomic sequence analysis is a very essential technique to explain whether morphologically similar sarcocysts from different intermediate hosts are identical species or not (Yang et al., 2001). The most common sequences reliable to discriminate Sarcocystis spp. are 18S rRNA, 28S rRNA, COX1, and ITS sequences (Bahari et al., 2014; Bittencourt et al., 2016; Whaeeb and Faraj, 2016; Hu et al., 2017; Elmishmishy et al., 2018; El-Morsey et al., 2019; Gjerde et al., 2020; Metwally et al., 2019). In Iraq, two studies were conducted (Whaeeb and Faraj, 2016; Dakhil et al., 2017) concerning the PCR sequencing and phylogenetic analysis of the 18S rRNA gene among sheep for $S$. tenella and water buffalos for S. fusiformis and S.moulei. There is no molecular and ultrastructural study on Sarcocystis spp. of sheep and goats in the Kurdistan region, Iraq. Therefore, the current study was designed to identify macroscopic Sarcocystis spp. in naturally infected domestic sheep and goats using phylogenetic analysis of $18 \mathrm{~S}$ rRNA sequences and 
to evaluate their genetic variants, as well as to study the morphological and the ultrastructural characteristics of the isolated species in Soran, Erbil, Iraq

\section{MATERIALS AND METHODS}

\section{Morphologic characterization of macrosarcocysts}

The entire esophagi were collected from 400 sheep and 600 goat carcasses at the slaughterhouse of Soran city, Erbil, Kurdistan, Iraq, from August 2018 to August 2019. The tissue samples were transported to the laboratory in icecooled boxes for further analysis. The esophagi were longitudinally sectioned to examine its internal and external walls (Bittencourt et al., 2016). The dimensions of the macrosarcocysts were measured by a ruler, and then several of them were selected and processed for the ultra-histological study, while other macrosarcocysts were kept at $-20^{\circ} \mathrm{C}$ until analyses with molecular methods. The microscopic detection was performed by cutting a small portion of the macrocyst, and then it was compressed between two slides, stained with Giemsa stain, and examined microscopically (Juyal et al., 1989).

For ultrastructure study by scanning electron microscopy (SEM), small portions of the macrosarcocysts were fixed with $2.5 \%$ glutaraldehyde in sodium cacodylate buffer for $24 \mathrm{~h}$ at $4{ }^{\circ} \mathrm{C}$, then washed in $0.1 \mathrm{M}$ cacodylate buffer. After that, the samples were post-fixed with $1 \%$ osmium tetroxide for $2 \mathrm{~h}$ at $4{ }^{\circ} \mathrm{C}$. The fixed specimens were dehydrated by sequential incubations in increasing concentrations of acetone $(35 \%, 50 \%, 75 \%, 95 \%$ for $10 \mathrm{~min}$, and $100 \%$ for 15 min. Finally, they were sputter-coated with gold and inspected by SEM (LEICA model CPD030, Germany) (Bittencourt et al., 2016; Metwally et al., 2019).

For transmission electron microscopy (TEM), small portions of macrosarcocysts from infected esophagi were fixed in $2.5 \%$ glutaraldehyde in sodium cacodylate buffer for $24 \mathrm{~h}$ at $4{ }^{\circ} \mathrm{C}$. Post fixation was done for $2 \mathrm{~h}$ in $1 \%$ osmium tetroxide. The specimens were dehydrated in a graded acetone series, infiltrated with 1:1 acetone and Epon resin mixture, and embedded in Epon resin. The resin was polymerized in an oven at $60{ }^{\circ} \mathrm{C}$ overnight. Sections of $700 \AA$ thick were prepared by LKB ultramicrotome with a diamond knife and stained with uranyl acetate and lead citrate. The stained sections were visualized under TEM (LEICA model CPD030, Germany) (El- Morsey et al., 2019).

\section{Molecular identification, sequencing, and phylogenetic analysis}

The DNA was extracted from 20 macrosarcocysts of both infected sheep and goats according to the manufacturer's instructions of the DNA extraction kit (FAVORGEN®, Cat. No. FATGK001A, Taiwan). Briefly, 25 mg of the macrosarcocyst sample was isolated, grounded by micro pestle in a microcentrifuge tube, and processed. Finally, the extracted DNA was stored at $-20{ }^{\circ} \mathrm{C}$ until further analysis. The DNA templates were prepared according to the primers manufacturer's protocol (GeNet Bio, South Korea). Lyophilized forward and reverse primers of Sarcocystis spp. 18S rRNA genes were prepared according to the manufacturer's instruction. The PCR was performed using the Sarcocystis spp. specific primers amplifying a portion of the $18 \mathrm{~S}$ rRNA gene as described in Table 1.

Table 1. Primers used for PCR amplification and sequencing of the $18 \mathrm{~S}$ rRNA gene of Sarcocystis spp. isolated from goats and sheep

\begin{tabular}{|c|c|c|c|}
\hline $\begin{array}{l}\text { Primers and } \\
\text { orientation }\end{array}$ & Sequence $\left(5^{\prime} \rightarrow 3^{\prime}\right)$ & $\begin{array}{c}\text { Amplicon size } \\
\text { (base pair) }\end{array}$ & References \\
\hline Primer 2L Forward & GGATAAACCGTGGTAATTCTATG & \multirow{2}{*}{850} & $\begin{array}{l}\text { Choi et al. (2018) } \\
\text { Kalantari et al. (2016) }\end{array}$ \\
\hline Primer 3H Reverse & GGCAAATGCTTTCGCAGTAG & & Calero-Bernal et al. \\
\hline Primer S1 Forward & GAATCCAAACCCCTTTCAGAGT & \multirow{2}{*}{1050} & \multirow{2}{*}{$\begin{array}{l}\text { Choi et al. (2018) } \\
\text { Formisano et al. (2013) }\end{array}$} \\
\hline Primer $1 \mathrm{H}$ Reverse & TATCCCCATCACGATGCATAC & & \\
\hline Primer Forward & CGCAAATACTATATCACTCG & \multirow[b]{2}{*}{690} & \multirow[b]{2}{*}{ Hussein et al. (2017) } \\
\hline Primer Reverse & CTAGAAACCAACAAAATAGA & & \\
\hline Primer Forward & CGAATGGCTCATTAAAACAG & \multirow[b]{2}{*}{570} & \multirow{2}{*}{ Hussein et al. (2017) } \\
\hline Primer Reverse & CCAACTACGAGCTTTTTAAC & & \\
\hline
\end{tabular}


The PCR reaction was performed by using the super cycler PCR system (Kyratec ${ }^{\circledR}$ Thermo Fisher, Germany) according to the manufacturer's protocol for 30-35 cycles consisted of an initial denaturation step at $94{ }^{\circ} \mathrm{C}$ for 2 min, followed by 35 cycles of denaturation at $94{ }^{\circ} \mathrm{C}$ for $20 \mathrm{~s}$, annealing at $60{ }^{\circ} \mathrm{C}$ for $10 \mathrm{~s}$ and extension at $72{ }^{\circ} \mathrm{C}$ for $25 \mathrm{~s}$, with a final extension step at $72{ }^{\circ} \mathrm{C}$ for $5 \mathrm{~min}$. The PCR products were visualized by electrophoresis on $1 \%$ agarose gel with a 100 DNA marker (Favorgen, Taiwan), and purified using Favorgen ${ }^{\circledR}$ Gel and PCR Clean-up (Favorgen, Taiwan). The purified amplicons were sent to South Korea for sequencing on both strands, forward and reverse. Positive PCR products were directly sequenced by using oligonucleotide primers for identifying specific DNA regions of Sarcocystis species.

Forward and reverse sequencing was performed using ABI Prism Big Dye Terminator Cycle Sequencing Ready Reaction Kit, version 1.1 (Macrogen Inc., South Korea). Sequenced fragments were purified and resolved by capillary electrophoresis with the 3730xl Genetic Analyzer (Macrogen Inc., South Korea). The resulting sequences were aligned with other sequences of Sarcocystis spp. from intermediate hosts deposited in the GenBank using the BLAST tool and subjected to phylogenetic analyses (Rubiola et al., 2019). The neighbor-joining method was used to create a guide tree as pairwise and multiple sequence alignment. The Sanger sequencing technique was used for sequencing purposes. Phylogenetic analyses were performed based on nucleotide sequences of the 18S rRNA gene and compared to Toxoplasma gondii as an outgroup parasite by using the MEGA7 program (Kumar et al., 2016). The bootstrap method was used by testing 1000 bootstrap replications.

\section{RESULTS}

\section{Morphologic characterization}

The macroscopic sarcocysts were detected in 9.1\% (91/1000) of the inspected esophagi of both sheep and goats. Two different shapes of macroscopic sarcocysts were detected (Figure 1), which were described as thick and thin sarcocysts. The thick sarcocysts were oval to sac-like shape with bluntly rounded ends and were found in sheep and goats. The other type was thin, slender (fusiform), elongate with pointed ends, and was observed in goats only. The size of the thick sarcocysts ranged from $4 \times 2.5$ to $8 \times 5 \mathrm{~mm}$. While the thin sarcocysts varied from $5 \times 2$ to $10 \times 3 \mathrm{~mm}$ in size. Bradyzoites were found in all digested macroscopic sarcocysts samples of both animals (Figure 1D). Based on the gross examination, shape, and size of the macroscopic sarcocysts, the thick macroscopic sarcocysts were tentatively identified to $S$ gigantea and $S$. moule, whereas the thin type was assigned to S. medusiformis.

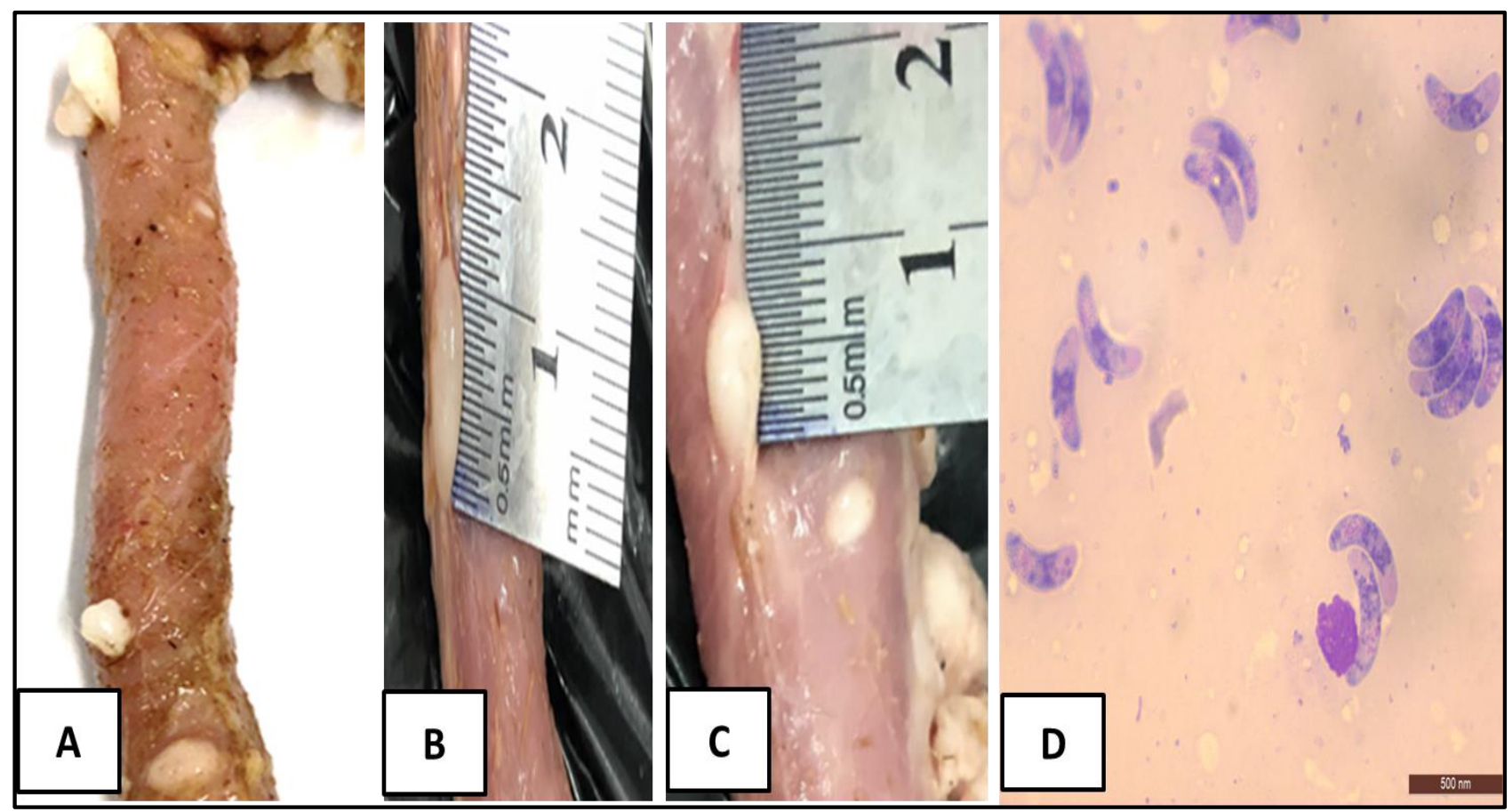

Figure 1. Gross appearance of the macroscopic sarcocysts in the esophageal muscles of infected sheep and goats with Sarcocystis spp. A: Oval shape sarcocyst in the sheep, B: Fusiform slender sarcocyst in the goats, C: Oval sac-like sarcocysts in the goats, D: Several banana-shaped bradyzoites from macroscopic sarcocyst stained with Giemsa stain $($ Scale bar $=500 \mathrm{~nm})$. 
The current results of SEM revealed three morphologically distinct sarcocysts types. Based on wall thickness, one of them was thin-walled $(<2 \mu \mathrm{m})$ without a clear secondary wall (Figure 2A), which preliminary was assigned to $S$. medusiformis. The second sarcocysts were thick-walled $(>5.0 \mu \mathrm{m})$ with secondary wall (Figure $2 \mathrm{~B}$ ) that was tentatively assigned to $S$. moulei, and the third one was thin-walled $(<2 \mu \mathrm{m})$ covered with connective tissue secondary wall that was provisionally referred to $S$. gigantea (Figure 3A). Sarcocysts were located within a parasitophorous vacuole in the host cell cytoplasm, consisting of a cyst wall that surrounds the metrocytes or the bradyzoites. Internally, groups of bradyzoites were separated into compartments by septa that arise from the sarcocyst wall as seen in Figure 2A, or they may not be clearly compartmentalized (Figure 2B).

The esophageal tissue appeared in both animals as a sheath covering the cyst with intact adhesion. Regarding the macroscopic sarcocyst which proposed belong to S. gigantea, it appeared with a thin wall surrounding by connective tissue secondary capsule as seen in Figure 3B. In one of the examined macroscopic sarcocysts of sheep, numerous recognizable bradyzoites appeared and looked like a banana in bundle shape resembles a rose (Figure 3C). There was no host tissue capsule surrounding the macroscopic sarcocyst of $S$. medusiformis (Figures $2 \mathrm{~A}$ and 4A), while $S$. gigantea had a host-derived connective tissue (Figure $3 \mathrm{~A}$ and B). This feature was considered as characteristic for macroscopic sarcocystis of $S$. medusiformis.

The present findings of TEM showed that there are ultrastructure similarities for all macroscopic sarcocysts sampled from sheep and goats. The sarcocysts were filled with a granular substance, merozoites, metrocytes, some intermediate stages between metrocytes and cyst merozoites, and typical cyst bradyzoites. The merozoites had all the organelles observed in the bradyzoites, except the rhoptries. The bradyzoites seemed to be banana-shaped and were arranged separately or tightly in packets separated by septa. The size of the bradyzoite reached $11 \times 2.6 \mu \mathrm{m}$, and had a double-membrane pellicle, and consisted of a conoid in one of the apices, numerous micronemes, two rhoptries, as well as a long, convoluted mitochondrion, subterminal nucleus, and several amylopectin granules (Figures 5 and 6). The apical complex, numerous micronemes, and up to two rhoptries were located in the anterior region. Inclusion bodies, including amylopectin, lipid, and electron-dense granules were found in the central region of the bradyzoites, and cell organelles, including the nucleus, were located at the posterior region. The micronemes were small in size and appeared ovoid or round in cross-section, and they were located towards the conoid.

Moreover, the metrocyte appeared with a nucleus, ground substance, amylopectin, and without micronemes. Some cysts had muscular tissues adhered to the external layer of the cyst wall. In other cysts, the host muscle tissue was completely absent.
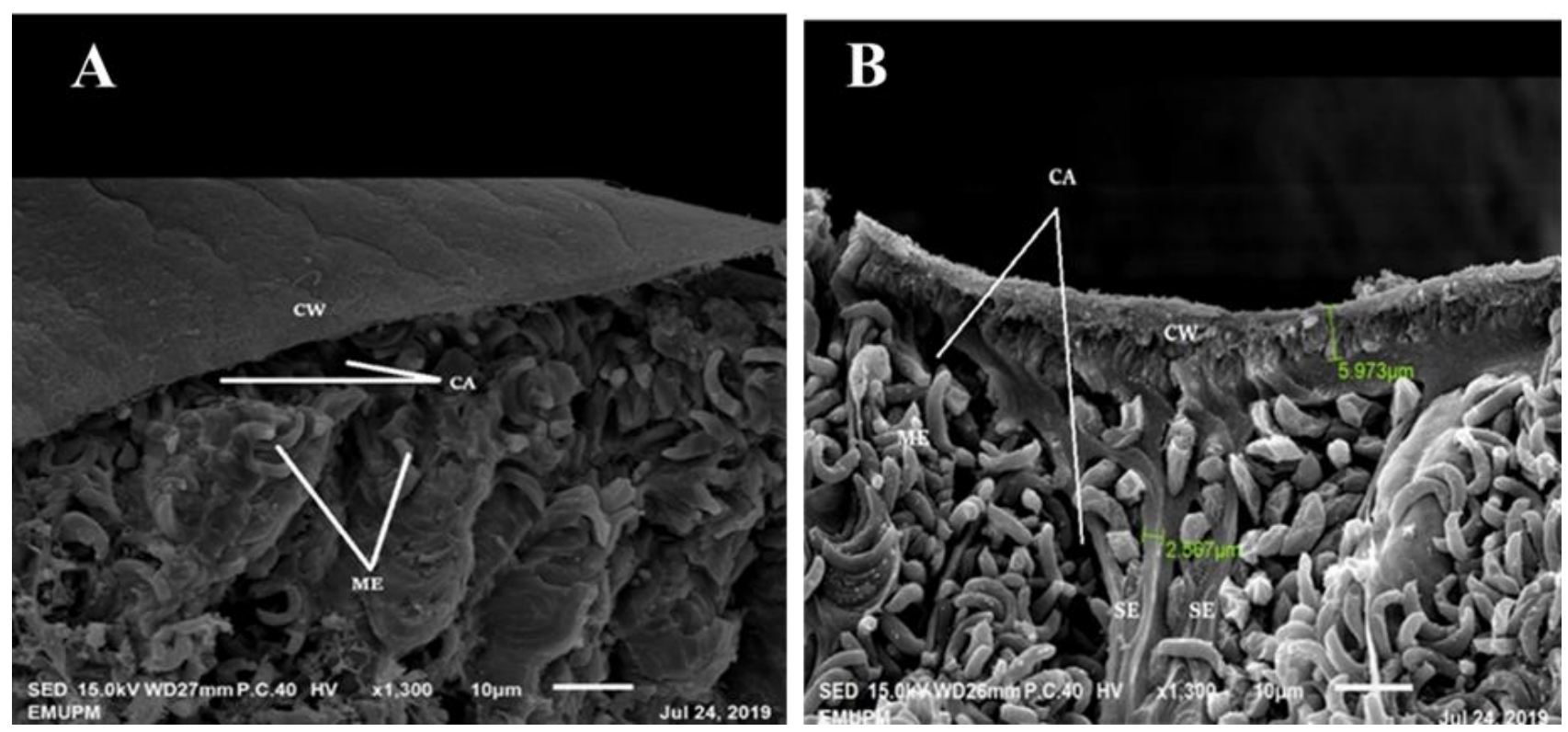

Figure 2. SEM of macroscopic sarcocyst in the esophagi of goat and sheep. A: S. medusiformis sarcocyst in goat. Note the groups of merozoite (bradyzoites), which appear like a banana shape in bundles or packed in chamber-like compartments separated from each other, and some chamber-like hollows (CA) between them. Scale bar= $10 \mu \mathrm{m}$. B: $S$. moule $i$ sarcocyst in sheep with the thick cell wall (CW), and filled with bradyzoites and metrocytes; the clear septa (Se) that originate from the sarcocyst wall like tree roots. Scale bar $=10 \mu \mathrm{m}$. 

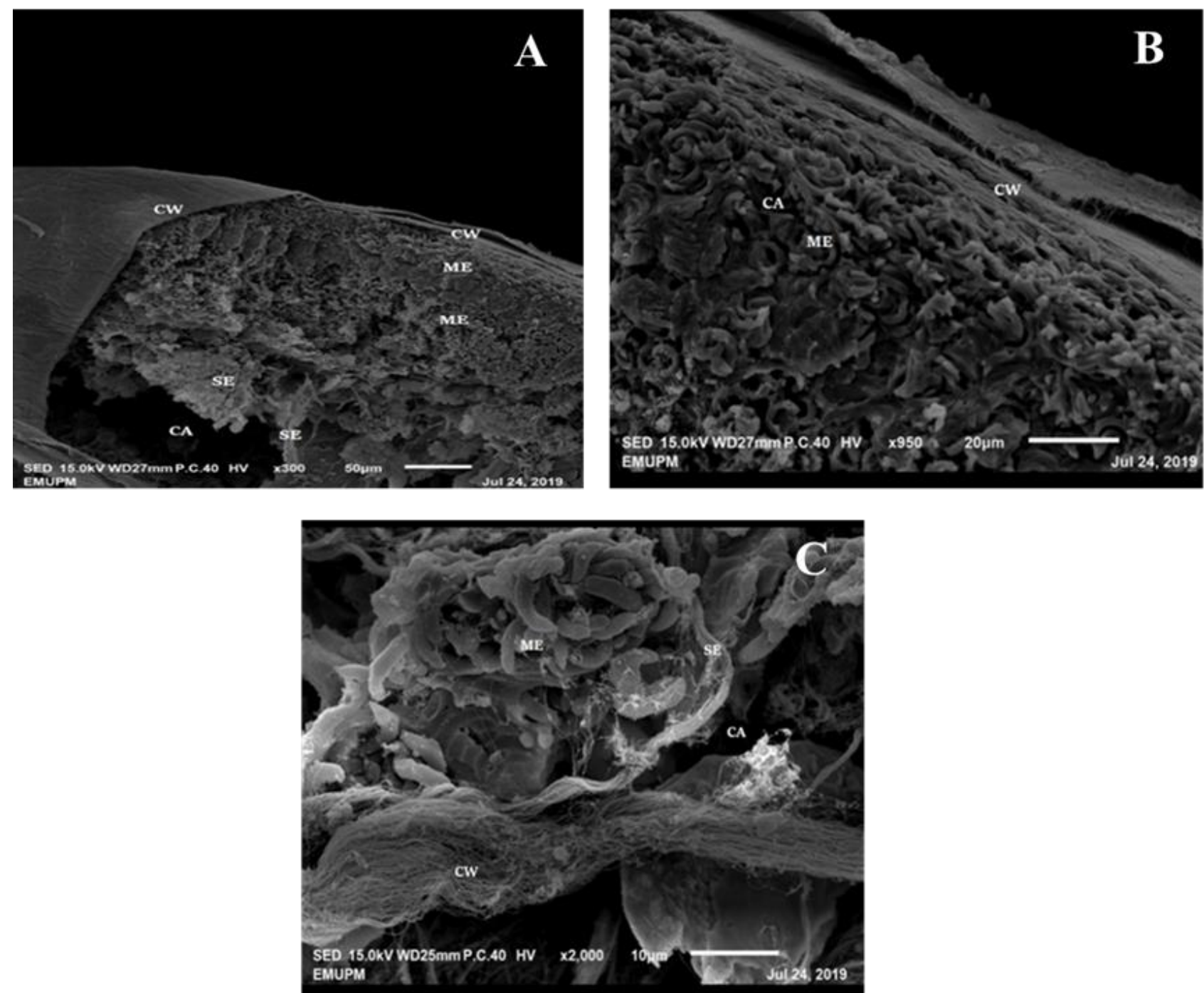

Figure 3. SEM micrograph of a macroscopic sarcocyst within the esophagi of sheep and goat. A: S. gigantea with thinwalled and highly patched merozoite (ME), the septa (SE) are thin; also, there is an appearance of chamber-like hollows (CA).Scale bar $=50 \mu \mathrm{m} ; \mathbf{B}$ : Peripheral site of macroscopic sarcocyst of S. gigantea with a thin wall surrounding connective tissue as a secondary capsule. Scale bar $=20 \mu \mathrm{m} ; \mathbf{C}$ : Part of sarcocyst showing the cell wall of a cyst with numerous obvious appearances of bradyzoites $(\mathrm{Br})$, which appear like a banana in bundle shape resembles a rose. Scale bar $=10 \mu \mathrm{m}$.
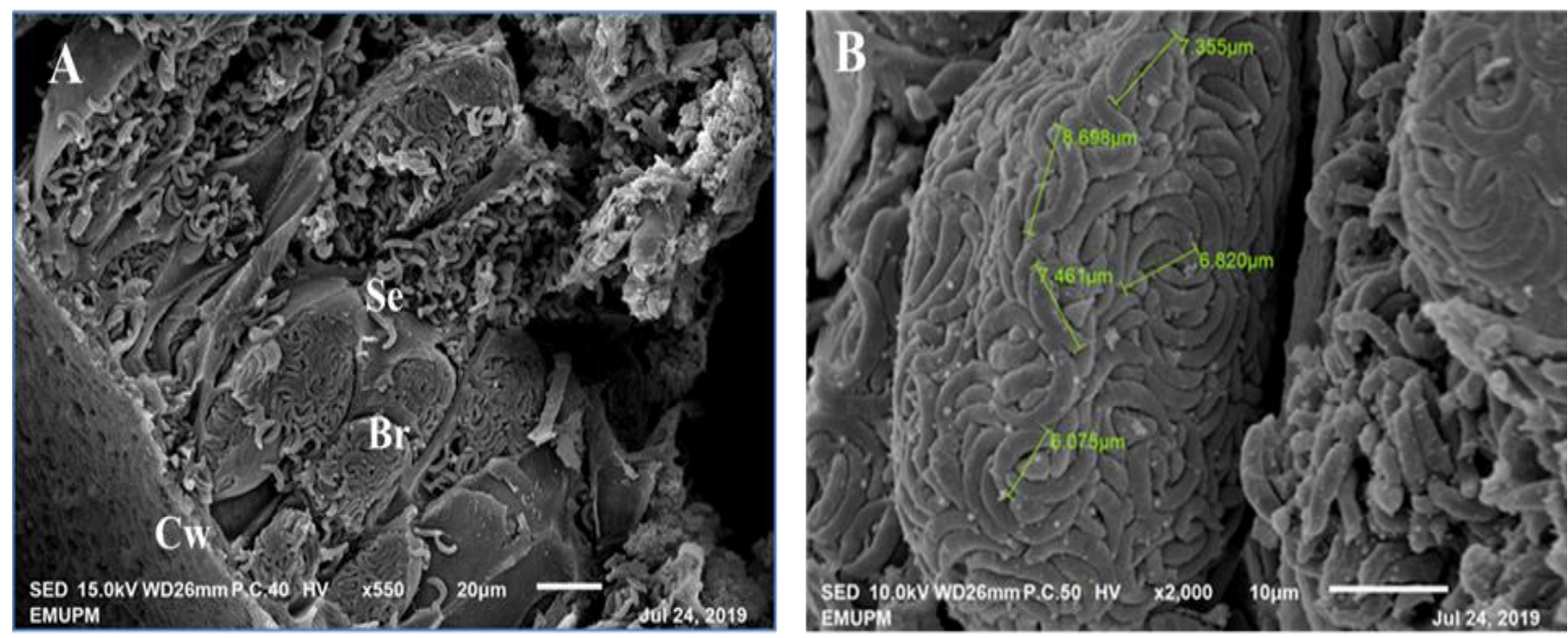

Figure 4. High magnification of macroscopic sarcocyst of S. medusiformis. A: Note the perforated cell wall (CW) and the numerous bundles of bradyzoites. Scale bar $=20 \mu \mathrm{m}$ B: Huge number of tightly packed bradyzoites of Sarcocystis in sheep ranged from 6 to $8.6 \mu \mathrm{m}$ in length. Scale bar $=10 \mu \mathrm{m}$. 

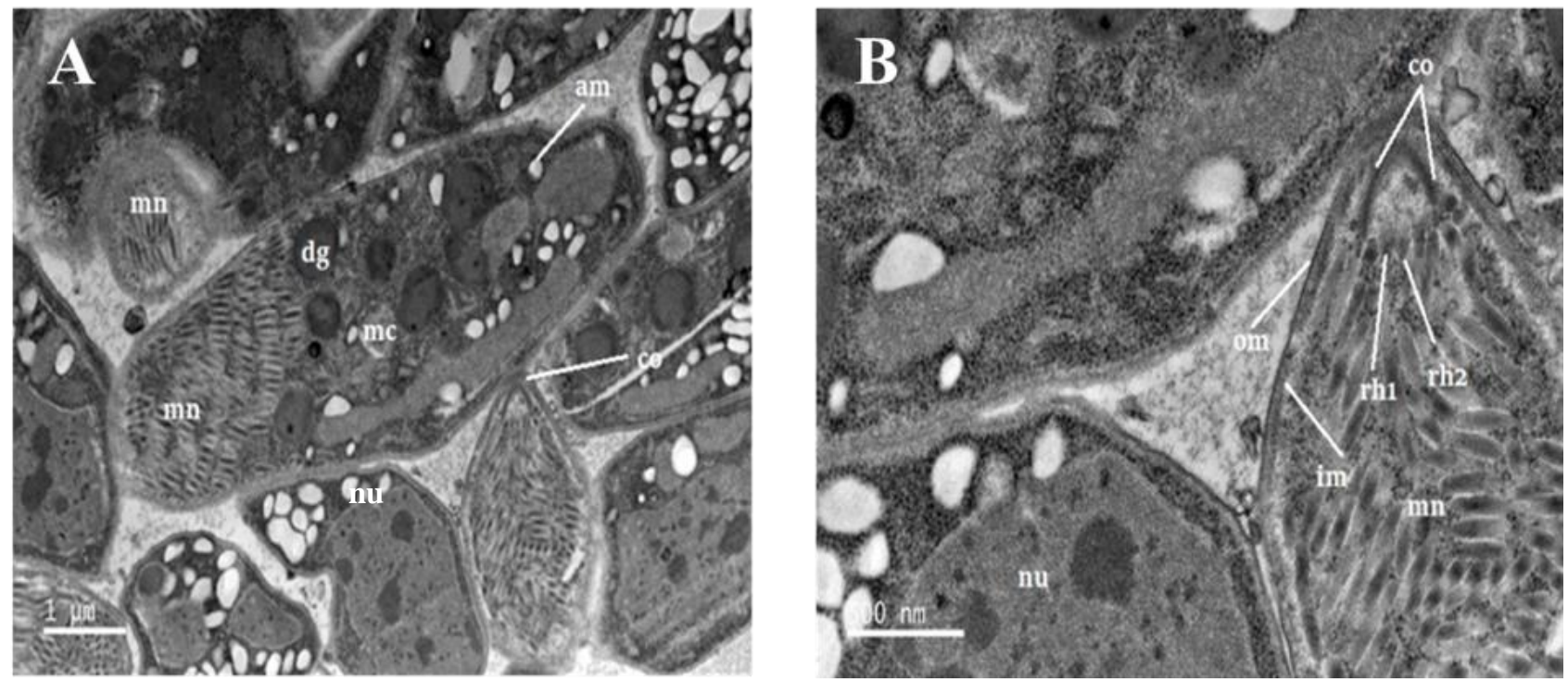

Figure 5. Transmission electron micrographs of a longitudinal section of macroscopic sarcocyst of Sarcocystis in goat. A: Merozoite of Sarcocystis; amylopectin (am), conoid (co), numerous micronemes (mn), nucleus (nu), convoluted mitochondrion (mc), dense granules (dg),Scale bar $=1 \mu \mathrm{m} ; \mathbf{B}$ : Conoidal (co) end of a bradyzoite of Sarcocystis with numerous micronemes (mn), rhoptries (rh), outer plasmalemma membrane (om), inner membrane (im), and tubular mitochondrion, Scale bar $=500 \mathrm{~nm}$.
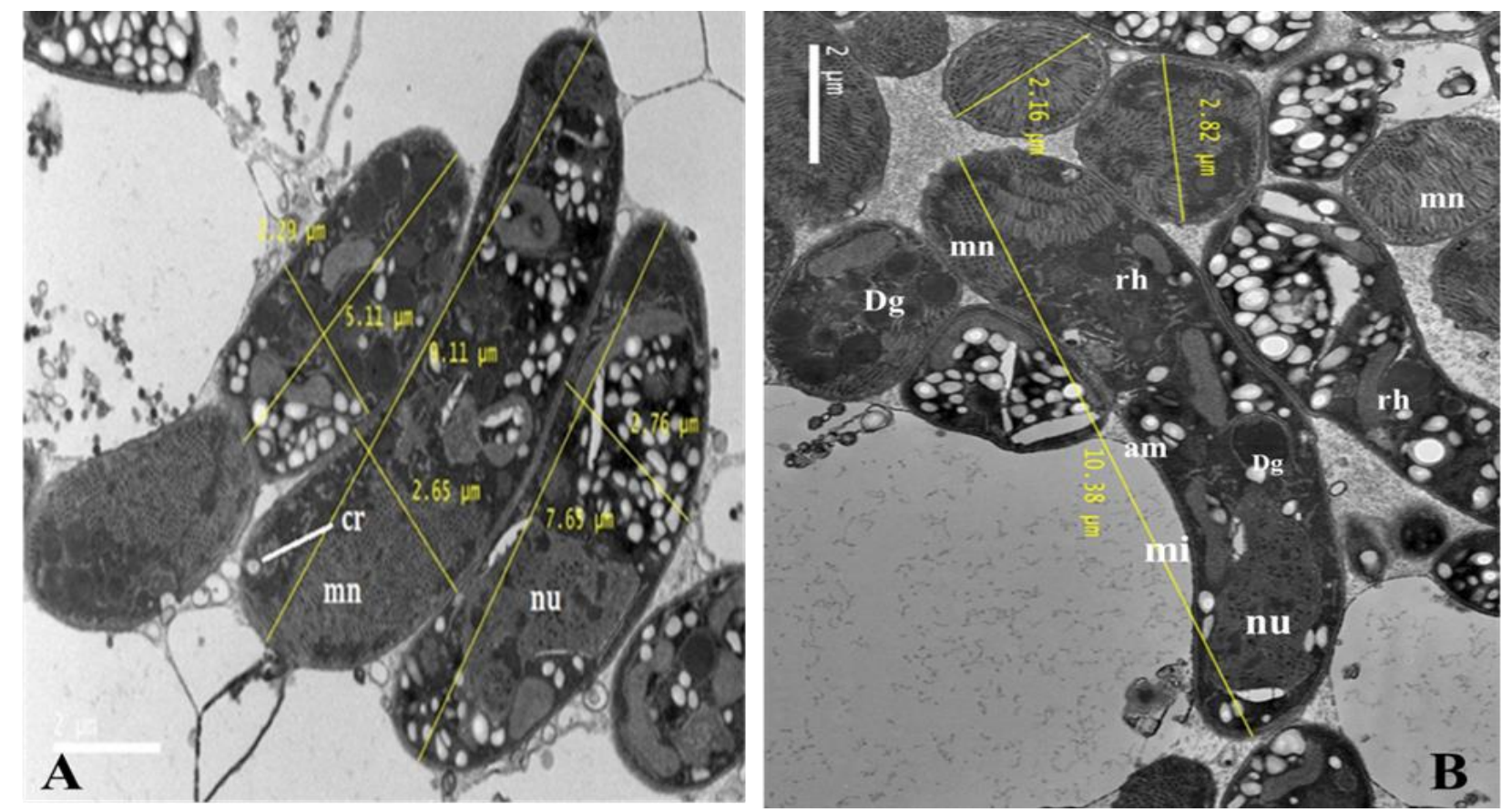

Figure 6. Ultrastructure of bradyzoites of sheep macroscopic sacocysyt of Sarcocystis. A: Note conoidal ring (Cr), amylopectin (am), numerous micronemes $(\mathrm{mn})$, nucleus $(\mathrm{nu})$. Scale bar=2 $\mu \mathrm{m}$. B: conoidal ring (cr), numerous micronemes (mn), nucleus ( $\mathrm{nu})$, rhoptries ( $\mathrm{rh}$ ), dense granules $(\mathrm{dg})$, amylopectin $(\mathrm{am})$, and mitochondrion (Mi) in sheep esophagus. Scale bar $=2 \mu \mathrm{m}$.

\section{Molecular characterization}

The PCR amplification revealed that all 20 isolates produced a positive band on gel electrophoresis with different amplicon molecular sizes. The partial 18S rRNA gene was amplified in all tested samples and yielded the expected amplicon PCR size of 1050, 850, 690, and 570 bp in both animals (Figure 7). Sanger sequencing was performed for identifying the Sarcocystis spp. by using forward and reverse primı.rs for eight macroscopic sarcocyst samples. The present findings proved that all macroscopic sarcocysts of both sheep and goat belonging to Sarcocystis spp and are deposited in the GenBank with accession numbers MN658377 from sheep and MN658381 from a goat. Comparison of one of the obtained sequences with other Sarcocystis spp. registered in GenBank was performed. The result of the phylogenetic analysis was compared with Toxoplasma gondii as out-group species and compared with Sarcocystis $18 \mathrm{~S}$ rRNA sequences from sequences deposited in the GenBank database. The eight sequences of Sarcocystis spp. were placed within the tree, (S1, S3, S4, G1, and G3) and (S2 and G2) were found in both sheep and goats, which related to $S$. gigantea and S. moulei, respectively. While G4 was recorded in goats and it was assigned to S. medusiformis (Figure 8). 
The sequencing of the amplified products resulted in 16 readable sequences, among those, S4 showed $99.43 \%$ identity with previously deposited sequence $S$. gigantean in GenBank with accession number MT026577 from Iran and $98.86 \%$ with $S$. moulei with the accession number of KC508513 from Iran. Also, S2 showed $99.46 \%$ identity with $S$. moulei under the accession number L76473 and $98.50 \%$ with S. gigantea with the accession number MK420020 from Spain. In addition, G3 showed $94.35 \%$ identity with S. gigantea of accession number KP053894 from Iran, Moreover, S1 showed $92.34 \%$ of homology with S. gigantea of the accession number MF769582, and G2 showed 94.66\% identity with the isolate of accession number L76473. While G4 display 92.61\% homology with S. medusiformis accession number MK42002.
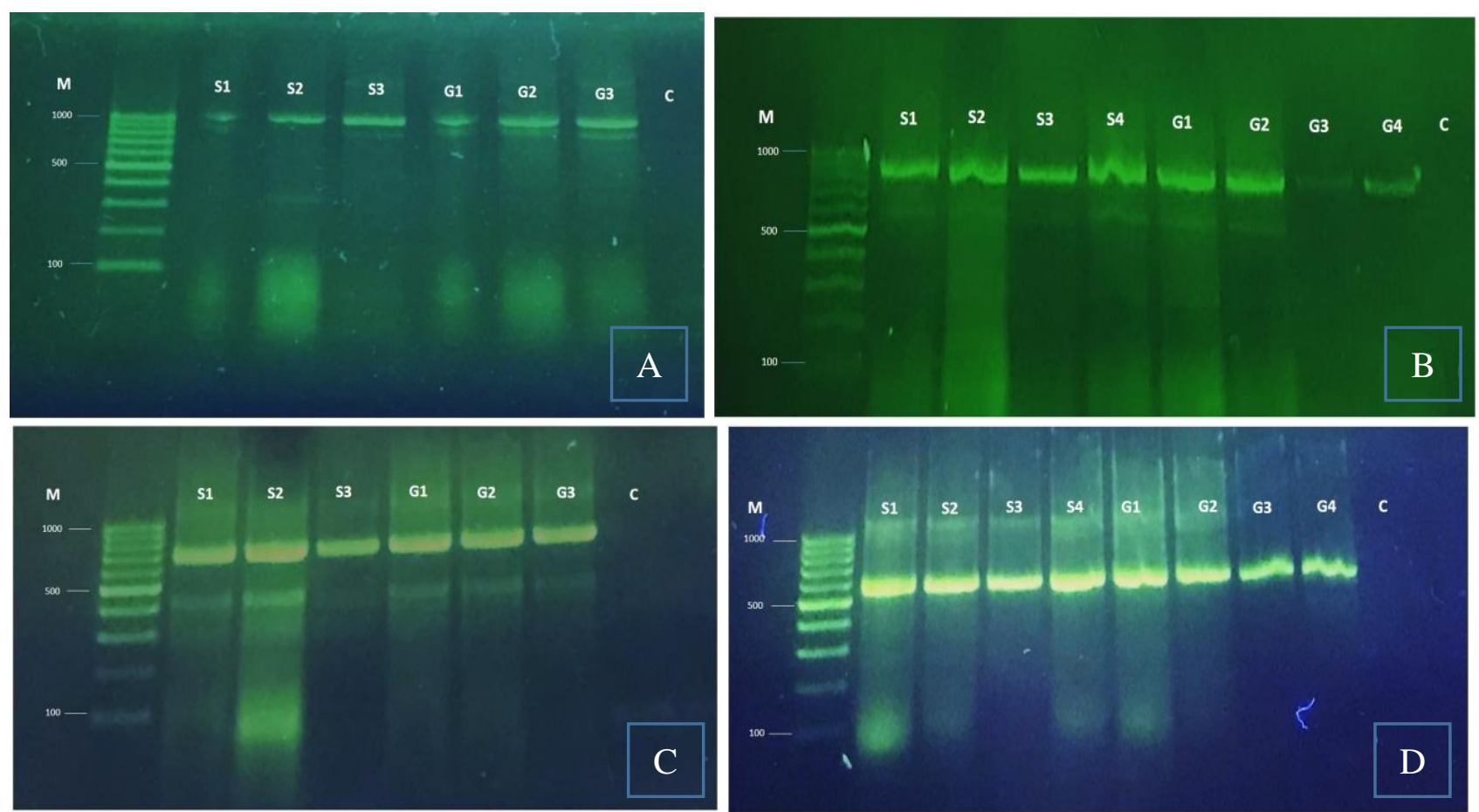

Figure 7. PCR products of partial 18S rRNA gene of Sarcocystis spp., presented bands at 1050 bp (A), 850 bp (B), 690 bp (C), and 570 bp (D) on 1\% agarose gel. M: 100 bp size marker. Lanes S1-S4: Sarcocystis spp. isolated from sheep. Lanes G1- G4: Sarcocystis spp. isolated from goats. Lane C: negative control.

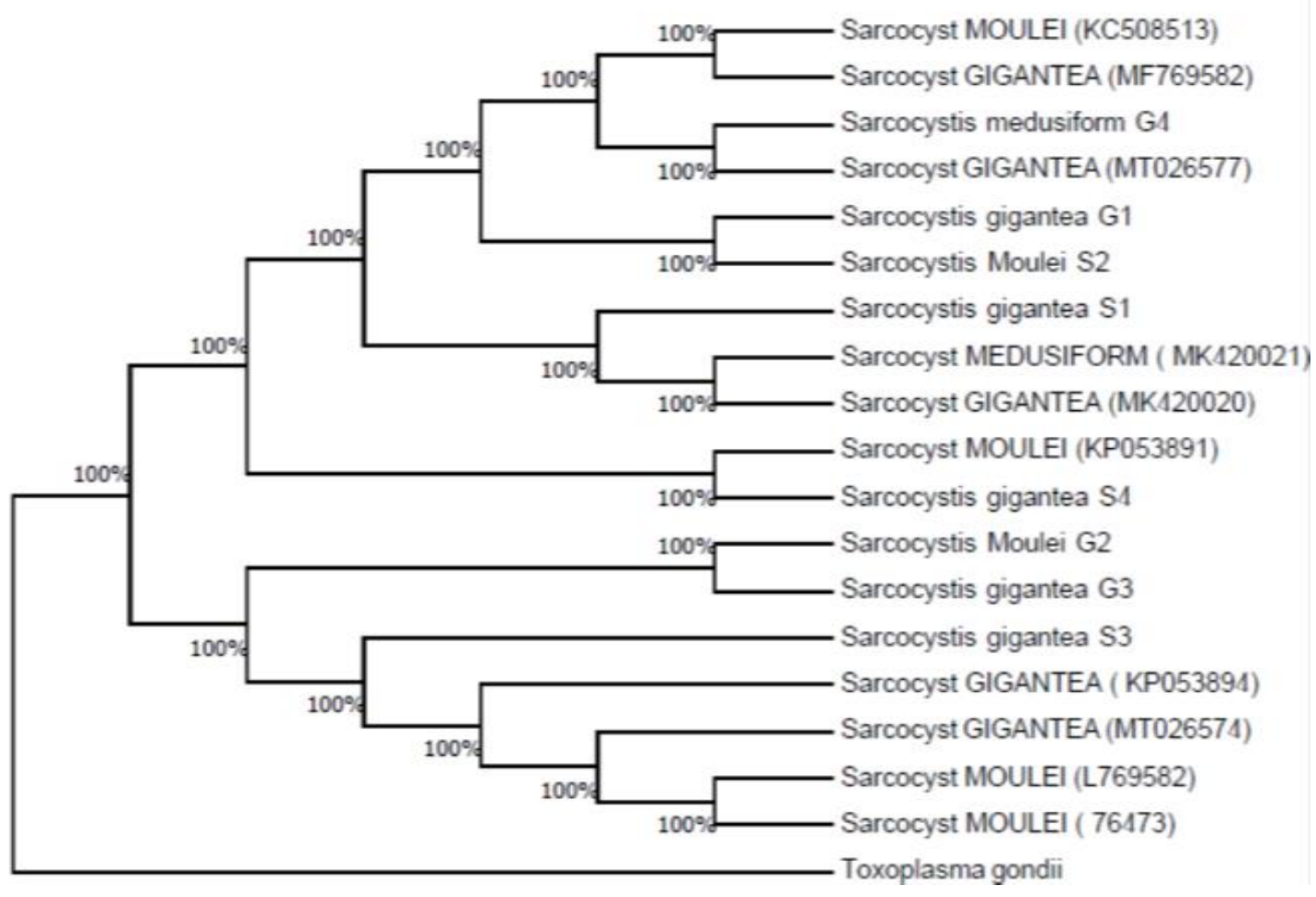

Figure 8. Phylogenetic tree of selected Sarcocystis spp by the program Mega v.7 using maximum likelihood-based on 18S r RNA gene sequences. The isolates of the present study represent as S1, S2, S3, S4, G1, G2, G3, and G4. Toxoplasma gondii was used as outgroup species. 
Sarcocystosis is prevalent in several ruminant livestock animals like cattle, sheep, and goats worldwide. The current study revealed that macroscopic Sarcocystis infection is common among sheep and goats. A large variation in the size and the shape of the sarcocysts was observed in the inspected esophagi of both animals, and this may be attributed to the age of the cyst as well as to the species of Sarcocystis.

Ultrastructural and molecular analysis of macroscopic sarcocysts showed three distinctive species of Sarcocystis. Regarding the sarcocysts from sheep origin, the results indicated that three of them represented S. gigantea, whereas the fourth one related to S.moulei. There are only two validated macroscopic species of Sarcocystis described in sheep; $S$. gigantea and S. medusiformis. S. gigantea is distributed worldwide, including Iraq (Al-Hyali et al., 2011), whereas $S$. medusiformis has only been reported from Italy, Iran, New Zealand, Spain, Jordan, and Australia (Collins et al., 1979; Dubey et al., 2016). The occurrence of $S$. moulei in sheep esophagi demonstrated that sheep can be a suitable host for S.moulei that is previously documented to parasitize goats. Elmishmishy et al. (2018) stated that there are very close phylogenetic interactions between S.gigantea and S.moulei, a goat specific species which rarely recorded in sheep and they suggested the cross-transmission between these two hosts. Moreover, a similar result was recorded in Iran and indicated that sheep can be a convenient and alternative host for S.moulei (Kalantari et al., 2016). Also, other researchers concluded that the Sarcocystis spp. of sheep and goats are highly related and should be together grouped (Metwally et al., 2019).

Concerning host specificity, investigation in Saudi Arabia identified S. moulei from sheep and goats (Al-Hoot et al., 2005), as well as a previous study, recorded the infection of goats with S. ovifelis (S. gigantea) and with $S$. moulei at the same time, besides, they concluded that the goats are the host for at least 3 Sarcocystis species that are hidden beneath the first description of S. moulei (Ghaffar et al., 1989). Also, a study from Iraq proved, for the first time, the infection of water buffalo with S.moulei in the world (Dakhil et al., 2017). The current work showed that the goats harbored three species of Sarcocystis, S. moulei, S. gigantea, and S. medusiformis. It is known that S. moulei is the macrosarcocysts of goats (Lindsay and Dubey, 2020).

The current study reported the first infection of goats with S. medusiformis in Iraq. This species was identified by morphological, ultrastructural, and molecular characterization, and in comparison with the formerly sequences that already existed in the GenBank of Sarcocystis spp. (Obendorf and Munday, 1987; Oryan et al., 1996; Gjerde et al., 2020). The morphologic characteristic of the sarcocysts of $S$. medusiformis as a thin fusiform shape and its size was consistent with the previous description of this species from sheep (Obendorf and Munday, 1987; Farhang-Pajuh et al., 2014). Besides, the muscle tissues infected with $S$. medusiformis were not encapsulated (Figure $3 \mathrm{~A}$, Figure 5A), but in the case of $S$. gigantea the muscle cell encapsulated by a host-derived connective tissue, but there was no real secondary wall (Figure 4A and B). This agreed with the findings of several studies (Munday and Obendorf, 1984; Obendorf and Munday, 1987; Ghaffar et al., 1989; Gjerde et al., 2020). The secondary cyst wall was detected in the old macrocyst of S.moulei; this agreed with the description of (Ghaffar et al., 1989), whereas in S. gigantea, there was no secondary wall, but there was a connective tissue capsule that originated from the host tissue. Furthermore, TEM results showed the typical structure of the bradyzoites, which contain the conoid, inner membrane complex, numerous micronemes, and rhoptries that are presented as electron-dense located at the anterior part. Also, there are dense granules scattered along the bradyzoites, particularly in the middle of it, tubular mitochondrion, amylopectin, and a sub-terminal large nucleus as described previously (Dubey et al., 2014; Dubey et al., 2016; El-Morsey et al., 2019).

In the present study, molecular detection was performed by using partial 18S rRNA genes for genotypic confirmation of the Sarcocystis spp. The variable regions of the 18S rRNA gene act as useful targets for the classification and characterization of dissimilar species (Neefs et al., 1991; Yang et al., 2001). Moreover, 18S rDNA is very convenient for phylogenetic investigations due to its high conservation, and examination of its variable regions permits the identification of species within a genus (Maidak et al., 1997; Ng et al., 2015). The PCR amplification of 20 macroscopic sarcocysts revealed that all isolates have a positive band on gel electrophoresis with different amplicons sizes. The partial 18S rRNA gene was amplified in all positive samples and yielded the expected amplicon PCR size of $850,1050,690$, and $570 \mathrm{bp}$ for both animals.

The current phylogenetic analysis was successfully generated with Toxoplasma gondii inferred from near fulllength 18S rRNA sequences and compared with previous sequences presented in GenBank. The occurrence of crossinfection may happen as seen in (S1, S3, S4, G1, and G3) belonged to S. gigantea in both sheep and goats, (S2 and G2) belonged to $S$. moulei, while $S$. medusiformis was only found in goat. Yang et al. (2001) concluded that morphologically similar species from two different intermediate hosts should be considered as the same species. Therefore, some Sarcocystis species seem to have a wider intermediate host range than formerly expected. The findings of the present study agreed with the outcomes of some previous investigations in this respect (Yang et al., 2001; Kalantari et al., 2016). The phylogenetic homogeneity between $S$ gigantea and $S$. moulei found in the current study can be reasoned by 
considering them as a sister clade, and this highlights the possibility of the occurrence of cross infection (Kalantari et al., 2016).

The current phylogenetic tree showed that the sequence of G4 from Iraqi goats assigned to S. medusiformis was highly similar to $S$. gigantea of accession number Mt026577. Similar findings were reported in a study related to Sarcocystis of sheep in Spain and showed that S. medusiformis was sister to sequence of S. gigantean (Gjerde et al., 2020). Moreover, the macrosarcocysts of S. medusiformis were recorded in Iran by two studies, the first one using a gross examination of the morphological features and transmission study, the shape of the isolated macrosarcocysts appeared narrow and elongate (Oryan et al., 1996), and the second by using the PCR-RFLP technique for the 18S rRNA gene, but they did not confirm the species by sequencing of the PCR product (Fahang-Pajuh et al., 2014). Besides, a recent study pointed out that S.capracanis of domestic goats is sister species of S.tenella of sheep (El-Morsey et al., 2019). The genotypic characteristic result agreed with the results obtained in Iran, Brazil, Argentina, and Spain (Bahari et al., 2014; Bittencourt et al., 2016; Gjerde et al., 2020). Nevertheless, it has documented that the mitochondrial COX1 sequence is considered to be a better marker than 18S rRNA, as COX1 gene could differentiate closely related Sarcocystis species in 18S rRNA analysis (Gjerde, 2013).

On the other hand, researchers compared the new sequences of the four genetic markers (18S rRNA, 28S rRNA, mitochondrial COX1, and ITS-1) for S. tenella and S. arieticanis, and confirmed that the ITS-1 region could be more useful for distinguishing closely related Sarcocystis spp owing to its high divergence (Hu et al., 2017). Also, ElMorsey et al. (2019) stated that the COX1 gene and the ITS- 1 sequences could be more accurate than the 18S rRNA and 28S rRNA genes for differentiating the closely related Sarcocystis spp. within the intermediate hosts because of their high divergence.

\section{CONCLUSION}

To the authors' knowledge, this is the first molecular and ultrastructural study of ovine and caprine Sarcocystis infection in Kurdistan, Iraq. The present findings showed that the isolated Sarcocystis spp. were most closely related to $S$. gigantea, S. moulei, and S. medusiformis and may consider them as sibling strains; the cross-infection may happen among sheep and goats, therefore, the host specificity of several Sarcocystis species is questionable. Further investigation with more precise gene markers like the mitochondrial COXI gene and the ITS-1 sequences are recommended to differentiate the closely related species, besides transmission study within the proposed definitive host is needed to provide further clarification of the biology of this enigmatic genus of protozoan parasites.

\section{DECLARATIONS}

\section{Authors' contributions}

Sara Omar Swar performed the practical work and wrote the draft of the manuscript. Bushra Hussain Shnawa conceived the idea, supervised the study, as well as a manuscript final reading. All the authors approved and agreed to publish the manuscript.

\section{Acknowledgments}

The authors gratefully acknowledge Dr. Chanar Najmaddin Abdullah from the Biology Department, Science College, Salahaddin University, for her help in drawing the phylogenetic tree.

\section{Competing interests}

The authors have declared that no competing interest exists.

\section{REFERENCES}

Al-Hoot AS , Al-Qureishy SA, Al-Rashid K, and Bashtar AR (2005). Microscopic study on Sarcocystis moulei from sheep and goats in Saudi Arabia. Journal of the Egyptian Society of Parasitology, 35(1): 295-312. Available at: https://europepmc.org/article/med/15881014

Al-Hyali NS, Kennany ER, and Khalil LY (2011). Fate of macrosarcocyst of Sarcocystis gigantea in sheep. Iraqi Journal of Veterinary Sciences, 25(2): 87-91. Available at: http://www.vetmedmosul.org/ijvs

Bahari P, Salehi M, Seydabadi M, and Mohammadi A (2014). Molecular identification of macroscopic and microscopic cysts of sarcocystis in sheep in North Khorasan province, Iran. International Journal of Molecular and Cellular Medicine, 3(1): 51-56. Available at: https://www.ncbi.nlm.nih.gov/pmc/articles/PMC3927390/

Barham M, Stutzer H ,Karanis P, Latif B, and Neiss W (2005). Seasonal variation in Sarcocystis species infections in goats in northern Iraq. Parasitology, 130(2): 151-615. DOI: https://doi.org/10.1017/S0031182004006134 
Bittencourt MV, Meneses IDS, Ribeiro-Andrade M, de Jesus RF, de Araújo FR, and Gondim LFP (2016). Sarcocystis spp. in sheep and goats: frequency of infection and species identification by morphological, ultrastructural, and molecular tests in Bahia, Brazil. Parasitology Research, 115 (4): 1683-1689. DOI: https://www.doi.org/10.1007/s00436-016-4909-5

Calero-Bernal R, Verma SK, Oliveira S, Yang Y, Rosenthal BM, and Dubey JP (2014). In the United States, negligible rates of zoonotic sarcocystosis occur in feral swine that, by contrast, frequently harbour infections with Sarcocystis miescheriana, a related parasite contracted from canids. Parasitology, 142(04): 549-556. DOI: https://www.doi.org/10.1017/s0031182014001553

Collins GH, Atkinson E, and Charleston WAG (1979). Studies on Sarcocystis species III: the macrocystic species of sheep. New Zealand Veterinary Journal, 27(10): 204-206. DOI: https://doi.org/10.1080/00480169.1979.34651

Dakhil HG, AbdallahB H, and Abdallah FA (2017). Molecular identification of Sarcocystis fusiformis and S. moulei infecting water buffaloes (Bubalus bubalis) in southern Iraq. World Journal of Pharmaceutical Research, 6(3): 215-229. DOI: https://doi.org/10.20959/wjpr20173-8013

Dubey JP, Calero Bernal R, Rosenthal BM, Speer CA, and Fayer R (2016). Sarcocystosis of animals and humans. 2nd Edn. Boca Raton: CRC Press; Taylor \& Francis Group, DOI: https://doi.org/10.1201/b19184

Dubey JP (2015). Foodborne and waterborne zoonotic sarcocystosis. Food and Waterborne Parasitology, 1(1): 2-11. DOI: https://doi.org/10.1016/j.fawpar.2015.09.001

Dubey JP, Lane EP, Van Wilpe E, Suleman E, Reininghaus B, Verma SK, Rosenthal BM, and Mtshali MS (2014). Sarcocystis cafferin. sp. (Protozoa: Apicomplexa) from the African Buffalo (Syncerus caffer). Journal of Parasitology, 100(6): 817-827. DOI: https://doi.org/10.1645/13467.1

Elmishmishy B, Al-Araby M, Abbas I, and Abu-Elwafa S (2018). Genetic variability within isolates of Sarcocystis species infecting sheep from Egypt. Veterinary Parasitology: Regional Studies and Reports, 13: 193-197. DOI: https://doi.org/10.1016/j.vprsr.2018.07.002

El-Morsey A, Abdo W, Sultan K, Elhawary NM, and AbouZaid AA (2019). Ultrastructural and Molecular Identification of the sarcocysts of Sarcocystis tenella and Sarcocystis arieticanis Infecting Domestic Sheep (Ovis aries) from Egypt. Acta Parasitologica, 64: 501-513. DOI: https://doi.org/ 10.2478/s11686-019-00070-8

Farhang-Pajuh F, Yakhchali M, and Mardani K (2014). Molecular determination of abundance of infection with Sarcocystis species in slaughtered sheep of Urmia, Iran. Veterinary Research Forum, 5(3): 181-186. Available at: https://www.ncbi.nlm.nih.gov/pmc/articles/PMC4279651/

Formisano P, Aldridge B, Alony Y, Beekhuis L, Davies E, Del Pozo J, and Beard PM (2013). Identification of Sarcocystis capracanis in cerebrospinal fluid from sheep with neurological disease. Veterinary Parasitology, 193, (1-3): 252-255. DOI: https://doi.org/ 10.1016/j.vetpar.2012.12.016

Ghaffar FA, Heydorn AO, and Mehlhorn H (1989). The fine structure of cysts of Sarcocystis moulei from goats. Parasitology Research, 75: 416-418. DOI: https://doi.org/10.1007/BF00931140

Gjerde B (2013) Sarcocystis species in red deer revisited: with a re-description of two known species as Sarcocystis elongatan. sp. and Sarcocystis truncata n. sp. based on mitochondrial sequences. Parasitology, 141(3): 441-452. DOI: https://doi.org/10.1017/s0031182013001819

Gjerde B, de la Fuente C, Alunda JM, and Luzón M (2020). Molecular characterization of five Sarcocystis species in domestic sheep (Ovis aries) from Spain. Parasitology Research. 119 (1): 215-231. DOI: https://doi.org/10.1007/s00436-019-06504-6.

Hong EJ, Sim C, Chae JS, Kim HC, Park J, Choi KS, Yu DH, Park CH, Yoo JG, and Park BK (2016). Ultrastructural and molecular identification of Sarcocystis tenella (Protozoa, Apicomplexa) in naturally infected Korean native goats. Veterinární Medicína, 61(7): 374-381. DOI: https://doi.org/10.17221/93/2015-vetmed

Hu J-J, Huang S, Wen T, Esch GW, Liang Y, and Li HL (2017). Sarcocystis spp. in domestic sheep in Kunming City, China: prevalence, morphology, and molecular characteristics. Parasite, 24: 30. DOI: https://doi.org/10.1051/parasite/2017025

Huang Z, Ye Y, Zhang H, Deng S, Tao J, Hu J, and Yang Y (2019). Morphological and molecular characterizations of Sarcocystis miescheriana and Sarcocystis suihominis in domestic pigs (Sus scrofa) in China. Parasitology Research, 118: 3491-3496. DOI: https://doi.org/10.1007/s00436019-06521-5

Hussein DE, Abu-Akkada SS, Bessat MS, Aggour MG, and Otify YZ (2017). Molecular identification of Sarcocystis species in imported frozen beef in Egypt. Alexandria Journal of Veterinary Sciences, 53 (2): 72-82. DOI: https://doi.org/10.5455/ajvs.244449

Juyal PD, Ruprah NS, and Chhabra MB (1989). Rapid isolation of intact micro-Sarcocystis (Protozoa-Apicomplexa) cysts from muscular tissue. Indian Journal of Animal Health, 28(1): 69-70.

Kalantari N, Khaksar M, Ghaffari S, and Hamidekish SM (2016). Molecular Analysis of Sarcocystis Spp. Isolated from Sheep (Ovis aries) in Babol area, Mazandaran Province. Northern Iran. Iran Journal of Parasitology, 11(1): 73-80. Available at: https://pubmed.ncbi.nlm.nih.gov/27095971/

Kumar S, Stecher G, and Tamura K (2016). MEGA7: Molecular evolutionary genetics analysis version 7.0 for bigger datasets. Molecular Biology and Evolution, 33(7): 1870-1874. DOI: https://doi.org/10.1093/molbev/msw054

Lindsay DS, and Dubey JP (2020). Neosporosis, Toxoplasmosis, and Sarcocystosis in Ruminants. The Veterinary Clinics of North America. Food Animal Practice, 36(1): 205-222. DOI: https://doi.org/10.1016/j.cvfa.2019.11.004

Maidak BL, Olsen GJ, Larsen N, Overbeek R, McCaughey MJ, and Woese CR (1997). The RDP (Ribosomal Database Project). Nucleic Acids Research, 25(1): 109-111. DOI: https://doi.org/10.1093/nar/25.1.109

Metwally DM, Al-Damigh MA, Al-Turaiki IM and El-Khadragy MF (2019). Molecular characterization of Sarcocystis species isolated from sheep and goats in Riyadh, Saudi Arabia. Animals, 9(5): 256. DOI: https://doi.org/10.3390/ani9050256

Munday BL, and Obendorf DL (1984). Morphology of Sarcocystis gigantea in experimentally-infected sheep. Veterinary Parasitology, 16(3-4): 193199. DOI: https://www.doi.org/10.1016/0304-4017(84)90036-0

Neefs JM, Van de Peer Y, Ruk PDE, Goris A, and Wachter RDE (1991). Compilation of small ribosomal subunit RNA sequences. Nucleic Acids Research, 19: 1987-2015.

DOI: https://www.doi.org/ 10.1093/nar/19.suppl.1987.

Ng YH, Fong MY, Subramaniam V, Shahari S, and Lau YL (2015). Short communication: Genetic variants of Sarcocystis cruzi in infected Malaysian cattle based on 18S rDNA. Research in Veterinary Science, 103: 201-204. DOI: https://www.doi.org/10.1016/j.rvsc.2015.10.009 
Obendorf DL and Munday BL (1987). Experimental infection with Sarcocystis medusiformis in sheep. Veterinary Parasitology, 24(1-2): 59-65. DOI: https://www.doi.org/10.1016/0304-4017(87)90130-0

Oryan A, Moghaddar N, and Gaur SNS (1996). The distribution pattern of Sarcocystis species, their transmission and pathogenesis in sheep in Fars Province of Iran. Veterinary Research Communications, 20(3): 243-253. DOI: https://www.doi.org/10.1007/bf00366922

Rubiola S, Chiesa F, Zanet S, and Civera T (2019). Molecular identification of Sarcocystis spp. in cattle: partial sequencing of Cytochrome C Oxidase subunit 1 (COI). Italian Journal of Food Safety, 7(4): 7725. DOI: https://doi.org/10.4081/ijfs.2018.7725

Whaeeb ST, and Faraj AA (2016). Molecular identification and phylogeny of microscopic Sarcocystis sheep in Baghdad Province. International Journal of Advanced Research in Biological Sciences, 3(12): 50-56. DOI: http://dx.doi.org/10.22192/ijarbs.2016.03.12.006

Yang Z, Zuo Y, Yao Y, Chen X, Yang G, and Zhang Y (2001). Analysis of the 18S rRNA genes of Sarcocystis species suggests that the morphologically similar organisms from cattle and water buffalo should be considered the same species. Molecular and Biochemical Parasitology, 115(2): 283-288. DOI: https://www.doi.org/10.1016/s0166-6851(01)00283-3 